\title{
EFFECTS OF SOIL MOISTURE, NPK FERTILIZERS AND VARIETIES ON GRAIN GROWTH OF BARLEY (Hordeum vulgare L.)
}

\author{
M. S. I. Mollah, M. H. Rashid1, M. S. Hossain' ${ }^{2}$ and M. Khalekuzzaman' \\ Institute of Biological Science (I.B.Sc), University of Rajshahi, Rajshahi-6205, Bangladesh
}

\begin{abstract}
The experiments are conducted in the experimental field of the Department of Botany, Rajshahi University during the winter season of 2005-2006 to study the effects of soil moisture and NPK fertilizers on grain growth of four barley varieties (Hordeum vulgare L.) following split-split plot design. The highest grain growth parameters like, spikelet number, spike dry weight, grain number and grain dry weight, spike relative growth rate and grain relative growth rate were observed in the $I_{2}$ treatment at different days after anthesis. $F_{3}$ treatment produced the highest spikelet number, spike dry weight, grain number, grain dry weight, spike relative growth rate and grain relative growth rate but the control produced the lowest values. BHL-3 produced higher spikelet number and grain relative growth rate. BL-1 produced higher spike dry weight, grain number, grain dry weight and spike relative growth rate.
\end{abstract}

Key words : Soil moisture, Fertilizers, Varieties, Barley, Grain growth

\section{NTRODUCTION}

Barley is an important cereal crop cultivated successfully in a wider range of climate. It is the fourth grain crop both in area and production in the world after maize, wheat and rice. In Bangladesh, it is grown as a minor crop (rabi crop) mainly in the northern part of the country. It ranks third after rice and wheat as supplementary food and fodder crop in Bangladesh (FAO, 2002). In the whole world, now barley is an important crop for direct human consumption and for animal feed. The most important uses of barley are grain feed to livestock and poultry. It is also used in preparing "chapaties" and sometimes barley is mixed with gram or wheat for preparing better quality chapaties. Barley grain can be is used to prepare 'Sattu' (Barley flour is mixed with sugar and water). Grain is also broken and roughly ground into pearl barley to be used in soup. Diluted soup made from barley is used to feed the infants as Horlicks, Ovaltine, Robinson's barley, Hamilton's barley flour, Pancake mix, Viva and Multova etc. These are baby foods, for which in Bangladesh, several industries and pharmaceutical companies have to import a large amount of barley grain and malt extract for manufacturing baby patient's food and medicine.

Barley is high in carbohydrates with moderate amounts of protein, calcium and phosphorus. It is the source of B-vitamins, such as thiamin, riboflavin and niacin. It is also

\footnotetext{
1 Department of Botany, University of Rajshahi, Rajshahi-6205, Bangladesh

2 Department of G Engineering and Biotechnology, University of Rajshahi, Rajshahi-6205,

Bangladesh
} 
very good source of dietary fiber. It has $4.5 \%$ soluble and $9 \%$ insoluble fiber. Fiber in barley is more soluble than those of oat and wheat. Barley flour contains $13.5 \%$ total dietary fiber, while wheat flour contains only 9\% total dietary fiber (BARI, 2003-2004). Huge amount of vitamin, protein and minerals are available in barley, which is useful to keep our body healthy and disease free (BARI, 2003-2004). Foods prepared from barley are useful for diabetic and high blood pressure patients. It is suggested that barley could be therapeutic diet for diabetic patient, a good diet for kidney patient and the referred diet after convalescence (Ikegami et al., 1991).

In spite of all these positive facts about barley, the barley cultivation trend in the country is consistently down word. The area and production of barley for the fiscal year 1991-92 were 40,000 acres and 10,000 tons, whereas, it is 3,000 areas and 1,000 tons in 2004-2005 respectively. Thus, it is needed to cultivate barley to popularize among the growers. Barley through a minor crop of the country can play an important role in enhancing the food security of the country. The areas, where it grows well may be brought under barley cultivation. Whereas wheat, maize or rice cannot be possible to cultivate profitably due to saline or stress environment should be brought under barley cultivation (BARI, 20032004).

Proper land preparation, optimum time of sowing, recommended doses of fertilizer and irrigation are not usually practiced to raise the crop production. Thus, the productivity of this crop is very poor compared to that of the other countries. There are many reasons for this and these are lack of well developed irrigation facilities, increased cost of irrigation and fertilizers, lack of stress tolerant high yielding varieties (HYVs) and lack of saline tolerant HYVs etc. Among these, water being extremely limited in most of the barley growing areas and essentially demands high efficiency in it's use.

Again, farmers of Bangladesh use different fertilizers indiscriminately without adequate information concerning actual soil requirements. A clear understanding on use of different doses of NPK fertilizers and their utilization efficiency are pre-requisite to avoid indiscriminate use of fertilizers.

However, very little work has been done in Bangladesh to develop package of improved management practices required to achieve higher yield of this crop. Thus, the objectives of this investigations were-

i) to study the effects of soil moisture and NPK fertilizers on grain growth of barley.

ii) to assess optimum irrigation frequencies and to find out economic and optimum dose of NPK fertilizes.

iii) to find out a suitable variety of barley with respect to grain growth for higher yield.

\section{MATERIALS AND METHODS}

The experiment was conducted in the experimental field of the Department of Botany, University of Rajshahi during the period from November, 2005 to March, 2006 with for 
barley varieties namely BARI Barley-1, BARI Barley-2, BHL-3 and BL-1. The soil of the field was silty loam, having pH 7.5 as well as $35 \%$ moisture at field capacity. The experiment was arranged in split-split plot design with three replications. Each replicated field was divided into three main plots for irrigation treatment. Each main plot was divided into four sub-plots for fertilizer treatment. Each sub-plot was lastly divided into four sub-sub plots for four varieties of barley. Three levels of irrigation treatments $(0,20$, and $40 \mathrm{~mm}$ as $\mathrm{I}_{0}, \mathrm{I}_{1}$ and $\mathrm{I}_{2}$, respectively) were adopted at every 30 days interval for three times during the growing period of the crop. Four levels of fertilizer treatments were used. NPK levels (kg/ha) for N as area were 0, 40, 80 and 120; for P as TSP were 0, 25, 50 and 75 and lastly for $\mathrm{K}$ as MP were $0,15,30$ and 45 . These recommended basal doses of fertilizers were applied as $F_{0}, F_{1}, F_{2}$ and $F_{3}$ at each split plot before sowing of seeds. Each split plot size was $4 \mathrm{~m} \times 1.8 \mathrm{~m}$, having a plot to plot distance $1 \mathrm{~m}$, replication to replication distance $2 \mathrm{~m}$, row to row $20 \mathrm{~cm}$, and plant to plant $5 \mathrm{~cm}$. Intercultural operations sere adopted as and when necessary.

For grain growth analysis, five grain harvests were done at equal interval of seven days after anthesis. For this, main spike per plant per treatment per variety per replication was selected, tagged with identifying marks to collect them at 7, 14, 21, 28 and 35 days after anthesis (DAA), At each harvest, the spikes were packed separately in labelled paper bags and were dried in oven for 72 hours at $85^{\circ} \mathrm{C}$. Various components of grain growth analysis were spikelet number, (SN), spike dry weight (SDW) in gm, grain number (GN) and grain dry weight (GDW) in gm, spike relative growth rate (Spike RGR) and relative growth rate (Grain RGR) were also determined from between two successive harvests of spike and grain growth stages by using the following formulae according to the classical technique of growth analysis (Radford, 1967).

$R G R=\frac{\log _{e} W_{2}-\log _{e} W_{1}}{t_{2}-t_{1}}$

Where, $W_{2}$ and $W_{1}$ are the spike or grain dry weights per spike (main tiller) at the later $\left(t_{2}\right)$ and the former $\left(t_{1}\right)$ grain growth harvest respectively.

Statistical analysis was carried out following to Gomez and Gomez, 1984.

\section{RESULTS AND DISCUSSION}

\section{Effect of irrigation}

Mean squares from the analysis of variance of Spikelet number, spike dry weight (g), grain number, grain dry weight (g), spike RGR $\left(\mathrm{gg}^{-1} \mathrm{day}^{-1}\right)$, grain RGR ( $\left.\mathrm{gg}^{-1} \mathrm{day}^{-1}\right)$ of four barley varieties as influenced by different soil moisture regimes and NPK fertilizers are shown in Table 1. The lowest spiketet number was produced by the non-irrigated plants. Irrigation increased the number of spikelets spike ${ }^{-1}$, thus the $\mathrm{I}_{2}$ treatment produced the highest spikelet number (46.188) (Table 2). This result is corroborated with okuyama and Igarashi (1990); Rahman et al. (2001); Haider (2002); Rahman (2004) in wheat. Irrigated plants had higher spike dry weight (g) than the rainfed plants. The rainfed plants had the 
lowest spike dry weight (0.534g) at 7 DNA. Rahman and Paul (1998) noticed similar findings in wheat. The highest number of grains spike ${ }^{-1}(35.813)$ was in the irrigated plants ( $\mathrm{I}_{2}$ treatment) and the lowest (21.563) was in the control ( $\mathrm{I}_{0}$ treatment). Similar results were also reported by Labuschagne and Van-Deventer (1992) and Rahman (2004) in wheat. The highest grain dry weight $(1.166 \mathrm{~g})$ was in the $\mathrm{I}_{2}$ treatment and the lowest was in the control $(0.309 \mathrm{~g})$. Similar results were also stated by Machado and Paulsen (2001); Haider (2002); Rahman (2004) in wheat. Rainfed condition resulted on the lowest RGR of spike $\left(0.025 \mathrm{gg}^{-1}\right.$ day-1$\left.^{-1}\right)$ at 14 DAA. I treatment had the highest RGR of spike $\left(0.079 \mathrm{gg}^{-1}\right.$ day $\left.^{-1}\right)$ at 7 DAA. Higher grain RGR was in the irrigated plants $\left(0.071 \mathrm{gg}^{-1} \mathrm{day}^{-1}\right)$ at 7 DAA than those in the rainfed plants $\left(0.021 \mathrm{gg}^{-1} \mathrm{day}^{-1}\right)$. This result is in agreement with Haider (2002) in wheat.

Table 1. Mean squares from the analysis of variance of Spikelet number, spike dry weight (g), grain number, grain dry weight (g), spike RGR (gg-1 day-1), grain RGR (gg-1 day-1) of four barley varieties as influenced by different soil moisture regimes and NPK fertilizers

\begin{tabular}{|c|c|c|c|c|c|c|}
\hline \multirow[t]{2}{*}{ Sources of variation } & \multirow[t]{2}{*}{ df } & \multicolumn{5}{|c|}{ Days After Anthesis ( DAA ) } \\
\hline & & 7 & 14 & 21 & 28 & 35 \\
\hline & & \multicolumn{5}{|c|}{ Spikelet number } \\
\hline Replication & 2 & $2126.8^{* *}$ & $2590.4^{* *}$ & $2605.7^{* *}$ & $2590.4^{* *}$ & $2590.4^{* *}$ \\
\hline Irrigation & 2 & 22.000 & 5.671 & 9.015 & 5.671 & 5.671 \\
\hline Error (a) & 4 & 5.083 & 10.734 & 14.078 & 10.734 & 10.734 \\
\hline Fertilizer & 3 & $216.1^{* *}$ & $171.1^{* *}$ & $178.5^{* *}$ & $171.1^{* *}$ & $171.1^{* *}$ \\
\hline $\mathrm{I}^{*} \mathrm{~F}$ & 6 & 5.963 & 14.921 & 8.890 & 14.921 & 14.921 \\
\hline Error (b) & 18 & 13.819 & 17.057 & 18.504 & 17.057 & 17.057 \\
\hline Variety & 3 & $3861.1^{* *}$ & $4082.0^{* *}$ & $4097.4^{* *}$ & $4082.0^{* *}$ & $4082.0^{* *}$ \\
\hline $\mathrm{I}^{*} \mathrm{~V}$ & 6 & 6.199 & 2.296 & 1.890 & 2.296 & 2.296 \\
\hline $\mathrm{F}^{*} \mathrm{~V}$ & 9 & $32.5^{* *}$ & $31.7^{* *}$ & $33.4^{* *}$ & $31.7^{* *}$ & $31.7^{* *}$ \\
\hline $\mathrm{I}^{*} \mathrm{~F}^{*} \mathrm{~V}$ & 18 & 2.546 & 2.213 & 2.098 & 2.213 & 2.213 \\
\hline \multirow[t]{2}{*}{ Error (c) } & 72 & 2.547 & 2.214 & 2.099 & 8.536 & 8.536 \\
\hline & & \multicolumn{5}{|c|}{ Spike dry weight $(\mathrm{g})$} \\
\hline Replication & 2 & $0.082^{* *}$ & $0.806^{* *}$ & $1.803^{* *}$ & $4.288^{* *}$ & $4.414^{* *}$ \\
\hline Irrigation & 2 & 0.000004 & 0.002 & 0.003 & $0.002^{*}$ & 0.004 \\
\hline Error (a) & 4 & 0.000002 & 0.003 & 0.006 & 0.000 & 0.003 \\
\hline Fertilizer & 3 & $0.005^{\star *}$ & $0.020^{*}$ & 0.010 & 0.024 & $0.045^{* *}$ \\
\hline $\mathrm{I}^{*} \mathrm{~F}$ & 6 & 0.0003 & 0.003 & 0.005 & 0.001 & 0.001 \\
\hline Error (b) & 18 & 0.0006 & 0.006 & 0.013 & 0.023 & 0.008 \\
\hline Variety & 3 & $2.102^{* *}$ & $6.296^{* *}$ & $10.488^{* *}$ & $12.124^{* *}$ & $11.396^{* *}$ \\
\hline $\mathrm{I}^{*} \mathrm{~V}$ & 6 & 0.0001 & 0.0004 & 0.002 & 0.001 & 0.001 \\
\hline $\mathrm{F}^{*} \mathrm{~V}$ & 9 & $0.003^{* *}$ & 0.013 & 0.008 & 0.021 & 0.016 \\
\hline $\mathrm{I}^{*} \mathrm{~F}^{*} \mathrm{~V}$ & 18 & 0.0001 & 0.002 & 0.001 & 0.001 & 0.0003 \\
\hline Error (c) & 72 & 0.001 & 0.009 & 0.017 & 0.031 & 0.014 \\
\hline
\end{tabular}


Table 1 (Contd.)

\begin{tabular}{|c|c|c|c|c|c|c|}
\hline \multirow[t]{2}{*}{ Sources of variation } & \multirow[t]{2}{*}{ df } & \multicolumn{5}{|c|}{ Days After Anthesis (DAA ) } \\
\hline & & 7 & 14 & 21 & 28 & 35 \\
\hline & & \multicolumn{5}{|c|}{ Grain number } \\
\hline Replication & 2 & $1293.9 * *$ & $1165.3^{* *}$ & $1128.2^{* *}$ & $931.0^{* *}$ & $864.8^{* *}$ \\
\hline Irrigation & 2 & $1.6^{* *}$ & 3.756 & $9.12^{*}$ & $7.9^{* *}$ & $10.5^{\star *}$ \\
\hline Error (a) & 4 & 0.063 & 1.663 & 0.908 & 0.063 & 0.203 \\
\hline Fertilizer & 3 & 53.291 & 73.326 & 114.673 & 150.265 & 154.890 \\
\hline$I^{*} \mathrm{~F}$ & 6 & 0.034 & 1.729 & 0.460 & 0.519 & 0.880 \\
\hline Error (b) & 18 & 25.211 & 28.755 & 47.552 & 54.440 & 60.873 \\
\hline Variety & 3 & $3242.4^{* *}$ & $2831.7^{* *}$ & $2717.4^{* *}$ & $2531.3^{* *}$ & $2457.8^{* *}$ \\
\hline $\mathrm{I}^{*} \mathrm{~V}$ & 6 & 0.243 & 1.756 & 1.265 & 0.144 & 0.602 \\
\hline $\mathrm{F}^{*} \mathrm{~V}$ & 9 & $5.31^{*}$ & 4.053 & $6.8^{* *}$ & $4.8^{* *}$ & $6.0^{* *}$ \\
\hline $\mathrm{I}^{*} \mathrm{~F}^{*} \mathrm{~V}$ & 18 & 0.423 & 1.710 & 0.885 & 0.111 & 0.343 \\
\hline \multirow[t]{2}{*}{ Error (c) } & 72 & 2.638 & 4.066 & 3.386 & 1.770 & 1.453 \\
\hline & & \multicolumn{5}{|c|}{ Grain dry weight (g) } \\
\hline Replication & 2 & $0.086^{* *}$ & $0.515^{\star *}$ & $0.674^{* *}$ & $1.558^{* *}$ & $2.718^{* *}$ \\
\hline Irrigation & 2 & 0.0001 & 0.0001 & 0.0011 & 0.0028 & $0.015^{* *}$ \\
\hline Error (a) & 4 & 0.0007 & 0.0001 & 0.0005 & 0.0002 & 0.0002 \\
\hline Fertilizer & 3 & $0.011^{* *}$ & $0.0021^{*}$ & 0.0126 & $0.105^{*}$ & $0.4228^{*}$ \\
\hline$I^{*} \mathrm{~F}$ & 6 & 0.0004 & 0.00004 & 0.0007 & 0.0007 & 0.0022 \\
\hline Error (b) & 18 & 0.0011 & 0.0006 & 0.0059 & 0.028 & 0.095 \\
\hline Variety & 3 & $1.131^{* *}$ & $1.851^{* *}$ & $2.522^{* *}$ & $4.438^{* *}$ & $6.609^{* *}$ \\
\hline$I^{*} \mathrm{~V}$ & 6 & 0.0003 & 0.00004 & 0.0006 & 0.0006 & 0.0016 \\
\hline $\mathrm{F}^{*} \mathrm{~V}$ & 9 & 0.0033 & 0.0003 & 0.0056 & 0.0206 & $0.039^{* *}$ \\
\hline$I^{*} F^{*} V$ & 18 & 0.0003 & 0.00004 & 0.0006 & 0.0001 & 0.0007 \\
\hline \multirow[t]{2}{*}{ Error $(\mathrm{c})$} & 72 & 0.0018 & 0.00091 & 0.0006 & 0.0110 & 0.0124 \\
\hline & & \multicolumn{5}{|c|}{ Spike RGR $\left(\right.$ gg-1 $^{-1}$ day $\left.^{-1}\right)$} \\
\hline Replication & 2 & $0.0042^{* *}$ & 0.0004 & $0.0014^{* *}$ & 0.0001 & \\
\hline Irrigation & 2 & 0.00006 & 0.00001 & 0.00002 & 0.00001 & \\
\hline Error (a) & 4 & 0.00008 & 0.00013 & 0.00005 & 0.00006 & \\
\hline Fertilizer & 3 & 0.00005 & $0.0004^{*}$ & 0.00016 & 0.00031 & \\
\hline $\mathrm{I}^{*} \mathrm{~F}$ & 6 & 0.00007 & 0.00008 & 0.00007 & 0.00002 & \\
\hline Error (b) & 18 & 0.00018 & 0.000 & 0.00011 & 0.00017 & \\
\hline Variety & 3 & $0.0029^{* *}$ & $0.0041^{* *}$ & $0.0056^{* *}$ & $0.0077^{* *}$ & \\
\hline $\mathrm{I}^{*} \mathrm{~V}$ & 6 & 0.00001 & 0.00006 & 0.00011 & 0.00001 & \\
\hline $\mathrm{F}^{*} \mathrm{~V}$ & 9 & $0.0004^{*}$ & 0.00015 & 0.00012 & 0.0002 & \\
\hline $\mathrm{I}^{*} \mathrm{~F}^{*} \mathrm{~V}$ & 18 & 0.00003 & 0.00006 & 0.00004 & 0.00001 & \\
\hline Error (c) & 72 & 0.00017 & 0.00090 & 0.00023 & 0.00034 & \\
\hline
\end{tabular}


Table 1 (Contd.)

\begin{tabular}{|c|c|c|c|c|c|c|}
\hline \multirow[t]{2}{*}{ Sources of variation } & \multirow[t]{2}{*}{ df } & \multicolumn{5}{|c|}{ Days After Anthesis (DAA ) } \\
\hline & & 7 & 14 & 21 & 28 & 35 \\
\hline & & \multicolumn{5}{|c|}{ Grain RGR (gg-1 day $\left.{ }^{-1}\right)$} \\
\hline Replication & 2 & $0.0092^{* *}$ & $0.0009^{* *}$ & $0.0013^{* *}$ & $0.0006^{* *}$ & \\
\hline Irrigation & 2 & 0.00004 & 0.000002 & $0.00004^{* *}$ & 0.00013 & \\
\hline Error (a) & 4 & 0.00010 & 0.000003 & 0.000001 & 0.00002 & \\
\hline Fertilizer & 3 & $0.00076^{*}$ & 0.000002 & $0.0006^{* *}$ & $0.0012^{* *}$ & \\
\hline$I^{*} \mathrm{~F}$ & 6 & 0.00011 & 0.000001 & 0.00002 & 0.00003 & \\
\hline Error (b) & 18 & 0.00024 & 0.000050 & 0.0002 & 0.0002 & \\
\hline Variety & 3 & $0.0175^{* *}$ & $0.0005^{* *}$ & $0.0013^{* *}$ & $0.0006^{* *}$ & \\
\hline $\mathrm{I}^{*} \mathrm{~V}$ & 6 & 0.00007 & 0.000001 & 0.00001 & 0.000001 & \\
\hline $\mathrm{F}^{*} \mathrm{~V}$ & 9 & 0.00039 & 0.00013 & $0.00018^{*}$ & 0.000095 & \\
\hline $\mathrm{I}^{*} \mathrm{~F}^{*} \mathrm{~V}$ & 18 & 0.00004 & 0.000001 & 0.000001 & 0.000021 & \\
\hline Error (c) & 72 & 0.00020 & 0.000001 & 0.000070 & 0.000074 & \\
\hline
\end{tabular}

Table 2. Effect of irrigation on mean values of spikelet number, spike dry weight (g), grain number, grain dry weight $(\mathrm{g})$, spike RGR $\left(\mathrm{gg}^{-1} \mathrm{day}^{-1}\right)$ and grain RGR (gg-1 day $^{-1}$ ) at different days after anthesis

\begin{tabular}{|c|c|c|c|c|c|c|c|c|c|c|}
\hline & \multicolumn{5}{|c|}{ Days after anthesis (DAA) } & \multicolumn{5}{|c|}{ Days after anthesis (DAA) } \\
\hline & 07 & 14 & 21 & 28 & 35 & 07 & 14 & 21 & 28 & 35 \\
\hline & \multicolumn{5}{|c|}{ Spikelet number } & \multicolumn{5}{|c|}{ Spike dry weight (g) } \\
\hline $\mathrm{I}_{0}(00 \mathrm{~mm})$ & 29.219 & 31.563 & 31.563 & 31.563 & 31.563 & 0.534 & 0.829 & 0.970 & 1.188 & 1.445 \\
\hline $\mathrm{I}_{1}(20 \mathrm{~mm})$ & 35.969 & 40.094 & 40.094 & 40.094 & 40.094 & 0.593 & 1.032 & 1.251 & 1.575 & 1.863 \\
\hline $\mathrm{I}_{2}(40 \mathrm{~mm})$ & 42.531 & 46.188 & 46.188 & 46.188 & 46.188 & 0.614 & 1.076 & 1.343 & 1.789 & 2.032 \\
\hline \multirow[t]{2}{*}{$\operatorname{LSD}(5 \%)$} & 1.285 & 1.867 & 2.139 & 1.867 & 1.867 & 0.001 & 0.033 & 0.044 & 0.010 & 0.031 \\
\hline & \multicolumn{5}{|c|}{ Grain number } & \multicolumn{5}{|c|}{ Grain dry weight (g) } \\
\hline $\mathrm{I}_{0}(00 \mathrm{~mm})$ & 25.438 & 23.563 & 22.406 & 21.688 & 21.563 & 0.309 & 0.411 & 0.511 & 0.592 & 0.699 \\
\hline $\mathrm{I}_{1}(20 \mathrm{~mm})$ & 31.000 & 28.500 & 26.594 & 25.031 & 24.844 & 0.367 & 0.493 & 0.584 & 0.709 & 0.856 \\
\hline $\mathrm{I}_{2}(40 \mathrm{~mm})$ & 35.813 & 33.656 & 32.000 & 30.281 & 29.875 & 0.392 & 0.618 & 0.744 & 0.951 & 1.166 \\
\hline \multirow[t]{2}{*}{ LSD (5\%) } & 0.143 & 0.735 & 0.543 & 0.145 & 0.257 & 0.015 & 0.004 & 0.013 & 0.013 & 0.008 \\
\hline & \multicolumn{5}{|c|}{ Spike RGR $\left(\mathrm{gg}^{-1}\right.$ day $\left.^{-1}\right)$} & \multicolumn{5}{|c|}{ Grain RGR (gg-1 day $\left.^{-1}\right)$} \\
\hline $\mathrm{I}_{0}(00 \mathrm{~mm})$ & 0.062 & 0.025 & 0.032 & 0.030 & & 0.047 & 0.034 & 0.022 & 0.021 & \\
\hline $\mathrm{I}_{1}(20 \mathrm{~mm})$ & 0.077 & 0.027 & 0.037 & 0.027 & & 0.048 & 0.026 & 0.026 & 0.025 & \\
\hline $\mathrm{I}_{2}(40 \mathrm{~mm})$ & 0.079 & 0.030 & 0.044 & 0.026 & & 0.071 & 0.025 & 0.032 & 0.028 & \\
\hline LSD (5\%) & 0.005 & 0.006 & 0.004 & 0.004 & & 0.006 & 0.001 & 0.001 & 0.001 & \\
\hline
\end{tabular}

\section{Effect of NPK fertilizers}

Fertilizer applied plants produced higher number of spikilet spike-1 than that of nonfertilized plants. $F_{3}$ treatment produced the highest spikelet number (49.875) and this 
number increased with the increase of NPK application (Table 3). Similar trend of the effects of fertilizer was noticed in barley by Alam (2003) and in wheat by Singh et al. (1992); Patel et al. (1995); Tarique (2003); Rahman (2004); Khaleque (2005). Higher spike dry weight $(2.446 \mathrm{~g})$ was produced by the fertilizer applied plants than that of the control. The non-fertilizer treatment resulted in the lowest spike dry weight $(1.143 \mathrm{~g})$ at 35 DAA. This result was also corroborated with Rahman (2004); Khaleque (2005) in wheat. $\mathrm{F}_{3}$ Fertilizer plants produced the highest number of grains spike-1 (33.417) and the lowest was in no fertilizer application treatment. Rahman (2004); Khaleque (2005) noticed similar findings in wheat. Higher grain dry weight $(\mathrm{g})$ was in the fertilizer treated plants than in the control. Increased grain dry weight $(\mathrm{g})$ was produced by increasing fertilizer levels. $\mathrm{F}_{3}$ treatment had the highest grain dry weight (1.347g). Haque (2000); Rahman (2004); khaleque (2005) reported similar findings in wheat. The highest spike RGR was obtained in the $\mathrm{F}_{3}$ treatment $\left(0.077 \mathrm{gg}^{-1}\right.$ day $\left.^{-1}\right)$ at 7 DAA and the lowest was in $\mathrm{F}_{1}$ treatment $(0.017$ gg $^{-1}$ day $\left.^{-1}\right)$. The lowest grain RGR was in the non-fertilized plants at all the DAA. Fertilizer treated plants had higher grain RGR than the control. The highest grain RGR $\left(0.049 \mathrm{gg}^{-1}\right.$ day $\left.^{-1}\right)$ was in the $F_{3}$ treatment. Rahman (2004) and Khaleque (2005) found similar findings in wheat.

Table 3. Influence of fertilizer on mean values of spikelet number, spike dry weight (g), grain number, grain dry weight $(\mathrm{g})$, spike RGR $\left(\mathrm{gg}^{-1} \mathrm{day}^{-1}\right)$ and grain RGR (gg-1 day $\left.^{-1}\right)$ at different days after anthesis

\begin{tabular}{|c|c|c|c|c|c|c|c|c|c|c|}
\hline & \multicolumn{5}{|c|}{ Days after anthesis (DAA) } & \multicolumn{5}{|c|}{ Days after anthesis (DAA) } \\
\hline & 07 & 14 & 21 & 28 & 35 & 07 & 14 & 21 & 28 & 35 \\
\hline & \multicolumn{5}{|c|}{ Spikelet number } & \multicolumn{5}{|c|}{ Spike dry weight (g) } \\
\hline $\mathrm{F}_{0}(000000)$ & 23.667 & 25.375 & 25.375 & 25.375 & 25.375 & 0.273 & 0.430 & 0.554 & 0.803 & 1.143 \\
\hline $\mathrm{F}_{1}(402515)$ & 31.501 & 37.125 & 37.125 & 37.125 & 37.125 & 0.516 & 0.937 & 1.055 & 1.370 & 1.542 \\
\hline $\mathrm{F}_{2}(805030)$ & 41.583 & 44.750 & 44.750 & 44.750 & 44.750 & 0.705 & 1.127 & 1.295 & 1.738 & 1.986 \\
\hline $\mathrm{F}_{3}(1207545)$ & 46.875 & 49.875 & 49.875 & 49.875 & 49.875 & 0.827 & 1.427 & 1.852 & 2.173 & 2.446 \\
\hline \multirow[t]{2}{*}{$\operatorname{LSD}(5 \%)$} & 1.840 & 2.044 & 2.129 & 2.044 & 2.044 & 0.011 & 0.034 & 0.062 & 0.075 & 0.130 \\
\hline & \multicolumn{5}{|c|}{ Grain number } & \multicolumn{5}{|c|}{ Grain dry weight (g) } \\
\hline $\mathrm{F}_{0}(000000)$ & 20.125 & 18.333 & 16.958 & 15.875 & 15.625 & 0.123 & 0.231 & 0.287 & 0.334 & 0.387 \\
\hline $\mathrm{F}_{1}(402515)$ & 26.208 & 24.417 & 22.792 & 21.583 & 21.583 & 0.322 & 0.426 & 0.526 & 0.614 & 0.742 \\
\hline $\mathrm{F}_{2}(805030)$ & 35.042 & 33.000 & 31.833 & 30.917 & 30.917 & 0.449 & 0.621 & 0.740 & 0.932 & 1.149 \\
\hline $\mathrm{F}_{3}(1207545)$ & 41.625 & 38.458 & 36.992 & 34.083 & 33.417 & 0.530 & 0.750 & 0.898 & 1.131 & 1.347 \\
\hline \multirow[t]{2}{*}{ LSD (5\%) } & 2.485 & 2.654 & 3.413 & 3.652 & 3.862 & 0.016 & 0.012 & 0.038 & 0.083 & 0.153 \\
\hline & \multicolumn{5}{|c|}{ Spike RGR $\left(\mathrm{gg}^{-1}\right.$ day $\left.^{-1}\right)$} & \multicolumn{5}{|c|}{ Grain RGR $\left(\mathrm{gg}^{-1}\right.$ day $\left.^{-1}\right)$} \\
\hline $\mathrm{F}_{0}\left(\begin{array}{llll}0 & 00 & 00\end{array}\right)$ & 0.065 & 0.036 & 0.053 & 0.048 & & 0.038 & 0.023 & 0.020 & 0.019 & \\
\hline$F_{1}\left(\begin{array}{lll}40 & 25 & 15\end{array}\right)$ & 0.066 & 0.017 & 0.037 & 0.017 & & 0.038 & 0.024 & 0.022 & 0.026 & \\
\hline$F_{2}(805030)$ & 0.067 & 0.020 & 0.040 & 0.021 & & 0.046 & 0.025 & 0.032 & 0.028 & \\
\hline $\mathrm{F}_{3}(1207545)$ & 0.077 & 0.036 & 0.023 & 0.018 & & 0.049 & 0.025 & 0.031 & 0.029 & \\
\hline LSD (5\%) & 0.007 & 0.006 & 0.005 & 0.006 & & 0.029 & 0.003 & 0.006 & 0.007 & \\
\hline
\end{tabular}




\section{Effect of varieties}

BHL-3 had the highest number of spikelet spike-1 among the varieties (41.250). BL-1 had the highest spike dry weight $(1.813 \mathrm{~g})$ as well as grain dry weight $(1.058 \mathrm{~g})$ at the last harvest. Again the highest grain number (28.333) was found in BL-1 at the first harvest. At first harvest interval, BL-1 had the highest spike RGR, whereas, the highest grain RGR was observed in BHL-3 (Table 4).

Table 4. Mean values of spikelet number, spike dry weight (g), grain number, grain dry weight (g), spike RGR $\left(\mathrm{gg}^{-1} \mathrm{day}^{-1}\right)$ and grain RGR $\left(\mathrm{gg}^{-1}\right.$ day $\left.^{-1}\right)$ at different days after anthesis as influenced by varieties

\begin{tabular}{|c|c|c|c|c|c|c|c|c|c|c|}
\hline & \multicolumn{5}{|c|}{ Days after anthesis (DAA) } & \multicolumn{5}{|c|}{ Days after anthesis (DAA) } \\
\hline & 07 & 14 & 21 & 28 & 35 & 07 & 14 & 21 & 28 & 35 \\
\hline & \multicolumn{5}{|c|}{ Spikelet number } & \multicolumn{5}{|c|}{ Spike dry weight (g) } \\
\hline BARI Barley-1 & 34.125 & 41.000 & 41.000 & 41.000 & 41.000 & 0.563 & 0.953 & 1.179 & 1.502 & 1.745 \\
\hline BARI Barley-2 & 34.625 & 38.000 & 38.000 & 38.000 & 38.000 & 0.588 & 0.992 & 1.171 & 1.510 & 1.807 \\
\hline BHL-3 & 39.500 & 41.250 & 41.250 & 41.250 & 41.250 & 0.577 & 0.976 & 1.198 & 1.512 & 1.752 \\
\hline BL-1 & 35.375 & 36.175 & 36.875 & 36.875 & 36.875 & 0.592 & 1.008 & 1.208 & 1.559 & 1.813 \\
\hline \multirow[t]{2}{*}{$\operatorname{LSD}(5 \%)$} & 0.750 & 1.041 & 0.987 & 0.987 & 0.987 & 0.017 & 0.044 & 0.061 & 0.083 & 0.055 \\
\hline & \multicolumn{5}{|c|}{ Grain number } & \multicolumn{5}{|c|}{ Grain dry weight (g) } \\
\hline BARI Barley-1 & 29.625 & 27.250 & 25.333 & 23.833 & 23.583 & 0.337 & 0.498 & 0.593 & 0.712 & 0.814 \\
\hline BARI Barley-2 & 30.125 & 27.875 & 26.417 & 25.292 & 25.208 & 0.373 & 0.506 & 0.606 & 0.736 & 0.902 \\
\hline BHL-3 & 30.833 & 28.176 & 26.500 & 24.792 & 24.417 & 0.346 & 0.506 & 0.609 & 0.730 & 0.851 \\
\hline BL-1 & 32.417 & 30.917 & 29.625 & 28.542 & 28.333 & 0.368 & 0.519 & 0.642 & 0.832 & 1.058 \\
\hline \multirow[t]{2}{*}{ LSD (5\%) } & 0.763 & 0.948 & 0.865 & 0.625 & 0.567 & 0.012 & 0.014 & 0.012 & 0.049 & 0.052 \\
\hline & \multicolumn{5}{|c|}{ Spike RGR (gg-1 day $\left.^{-1}\right)$} & \multicolumn{5}{|c|}{ Grain RGR (gg-1 day-1) } \\
\hline BARI Barley-1 & 0.072 & 0.031 & 0.037 & 0.025 & & 0.060 & 0.027 & 0.025 & 0.020 & \\
\hline BARI Barley-2 & 0.072 & 0.023 & 0.040 & 0.030 & & 0.049 & 0.028 & 0.026 & 0.028 & \\
\hline BHL-3 & 0.073 & 0.030 & 0.035 & 0.025 & & 0.057 & 0.029 & 0.022 & 0.020 & \\
\hline BL-1 & 0.075 & 0.026 & 0.040 & 0.024 & & 0.055 & 0.030 & 0.032 & 0.031 & \\
\hline LSD (5\%) & 0.006 & 0.006 & 0.007 & 0.009 & & 0.007 & 0.000 & 0.004 & 0.004 & \\
\hline
\end{tabular}

From the present study, it is revealed that-

i) Increasing levels of soil moisture and NPK fertilizers influenced to increase the different characters of grain. BHL-3 produced the highest spikelet number (SN) and grain RGR and BL-1 produced the highest spike dry weight $(\mathrm{g})$, grain number, grain dry weight $(\mathrm{g})$ and spike RGR.

ii) Among the four varieties of barley, BL-1 was highest in grain growth at the highest level of irrigation and fertilizer than that of control (Mollah, 2007).

iii) If the soil moisture and fertilizer levels are maintained properly, better grain growth and higher grain yield can be achieved from the variety BL-1. 


\section{REFERENCES}

Alam, M. Z. 2003. Influence of planting dates and nitrogen levels on growth, grain yield and nitrogen utilization of barely (Hordeum vulgare L). Ph. D. Thesis, Crop Physiology Laboratory, Department of Botany, Rajshahi University, Rajshahi. pp. 61-292.

BARI (Bangladesh Agricultural Research Institute). 2003-2004. Barley Improvement. Annual Research Report. Plant Breeding Division. Joydebpur, Gazipur. p. 1.

FAO, 2002. FAOSTAT: Statistics database (online.) (Subset production within Agriculture database) Available at http:11apps.fao.org

Gomez, K. A. and Gomez, A. A. 1984. Statistical procedure for agricultural research. John wiley and sons. New York.

Haider, S. A. 2002. Effect of water stress on the physiology and grain yield of four bread wheat (Triticum aestivum L.) cultivars. Ph.D. Thesis, Crop Physiology Laboratory, Department of Botany, Rajshahi University. Rajshahi. pp. 71-342.

Ikegami, S., Tsuchihashi, F., Nakamura, K. and Innami, S. 1991. Effects of barley on development of diabetes in rats. J. Jap. Soc. Nut. Food Sci. 44 (6): 447-454.

Khaleque, M. A. 2005. Study on growth and yield of modern wheat varieties in different agronomic management practices. Ph. D. Thesis, Institute of Biological Sciences, Rajshahi University. Rajshahi. pp. 53-243.

Labuschange, M. T. and Van- Deventer, C. S. 1992. The effect of moisture stress on the yield and yield components of several wheat cultivars and their $\mathrm{F}_{1}$ hybrids. J. Agron. Crop Sci. 168(3): 153-158.

Machado, S. and Paulsen, G. M. 2001. Combined effects of drought and high temperature on water relations of wheat and sorghum. Plant and Soil. 233(2): 179-187.

Mollah, M. S. I. 2007. Studies on the physiological attributes and there relationship to yield of barley (Hordeum vulgare L) under different soil moisture regime and NPN fertilizers. Ph.D. Thesis, Crop Physiology Laboratory, Department of Botany, Rajshahi University, Rajshahi. pp. 115-156, 168-194.

Okuyama, L. A. and Igarashi, S. 1990. Grain yield and yield components of wheat as a function of water stress at tillering stage. Informa-Da-Pesquisa-Instituto-Agronomic Da-Parana. 94: 49-52.

Patel, N. M., Sadarja, S. G., B. B and Khanpara, V. D. 1995. Effect of nitrogen, potassium and Zinc on growth and yield of wheat (Triticum aestivum). Indian J. Agron. 40 (2): 290-292.

Radford, P. J. 1967. Growth analysis formulae-their use and abuse, Crop Sci. 7: 171-175.

Rahman, M. A. 2004. Effect of irrigation on nitrogen uptake, yield attributes of wheat varieties. Ph.D. Thesis, Crop physiology Laboratory, Department of Botany, Rajshahi University. pp. 45-257.

Rahman, M. S. and Paul, N. K. 1998. Effect of soil regimes on physiological characters and yield of wheat cultivars. J. Bio. Sci. 6: 5-10.

Rahman, M. S., Sarker, A. M. Islam, M. S. and Paul, N. K. 2001. Effect of Soul mosture on grain yield of wheat (Triticum aestivum L.) cultivars. Environment $\mathcal{E}$ Ecol. 19 (2): 304-308.

Singh, R. V., Dubey, V. K. and Vyas, M. D. 1992. Effect of Seed rate, nitrogen level and method of fertilizer placement on wheat (Triticum aestivum) under the sown condition. Indian J. Agron. 37(1): 43-46.

Tarique, M. H. 2003. Growth and yield of wheat (Triticum aestivum L.) in relation to NPK fertilizers and competition. Ph.D. Thesis, Crop Physiology Laboratory, Department of Botany, Rajshahi University, Rajshahi. pp. 51-145, 149-258. 